\section{Surgical management of upper lid epiblepharon}

\begin{abstract}
Background Upper lid epiblepharon refers to a developmental anomaly characterised by the presence of an exaggerated skin fold running horizontally across the upper lid below the lid margin, which in severe cases is associated with lash-cornea touch or even corneal punctate epithelial erosions.

Method This retrospective study looked at 14 patients who underwent bilateral upper lid pretarsal orbicularis debulking and skin excision to correct lash-globe contact from the upper lid.
\end{abstract}

Results Surgery was successful in 25 of 28 lids, with one recurrence being successfully re-operated. The remaining 2 recurrences did not undergo further surgery as the patients were asymptomatic.

Conclusion This surgical procedure appears to be an effective treatment of lash inversion in upper lid epiblepharon with good aesthetic results.

Key words Anterior lamella, Epiblepharon, Trichiasis, Upper lid

Epiblepharon ${ }^{1}$ is commonly encountered in Oriental infants and children. In most cases it resolves with age, but persistence into adulthood is not uncommon and this can commonly be misdiagnosed as an upper lid entropion or ptosis. In entropion, however, the entire lid margin is turned against the globe, while in upper lid epiblepharon the lid margin is normal in position but the lashes project straight down or are turned inwards towards the cornea by a fold of skin - a feature that is aggravated on upgaze. Upper lid epiblepharon also should not be confused with ptosis, as the upper lid margin in the former condition is at the normal height. It is important to differentiate these three conditions as their surgical management is quite different.

The aetiology of upper lid epiblepharon has been attributed to a Z-shaped kink in the orbicularis fibres ${ }^{2}$ or the absence of levator aponeurotic attachment to the orbicularis and skin. ${ }^{3}$ It is known that Orientals have an exaggerated upper lid skin fold and a poor lid crease. This is due to a lower insertion of the orbital septum onto the levator aponeurosis. ${ }^{4}$ In the presence of a weak or absent insertion of the levator fibres onto the skin and orbicularis, the anterior lamella of the lid overhanging the lid margin causes the eyelashes to be turned straight down (lash ptosis) or inwards towards the cornea (Figs. 1,4). The tarsal position is normal. Removal of this overhanging anterior lamella and repositioning it should correct the lash ptosis in upper lid epiblepharon.

\section{Subjects and methods}

The case notes of 14 consecutive patients who underwent upper lid epiblepharon surgery between November 1994 and January 1996 were reviewed. All patients had bilateral upper lid epiblepharon with lash ptosis or trichiasis with corneal punctate epithelial erosions. They had had no previous eyelid surgery.

Pre-operative data collected included age, sex, race and documentation of the symptoms (Table 1). The operations were performed by one surgeon. Local anaesthesia was used except when the patient was a child.

\section{Surgical techniques}

The amount of redundant upper eyelid skin was assessed and drawn on the closed lid while gently pinching the skin using a pair of forceps. Care was taken to avoid causing post-operative ectropion from excessive skin excision. The skin crease level was set by placing the skin incision $5 \mathrm{~mm}$ above the lid margin. The skin ellipse was drawn on the skin before $2-3 \mathrm{ml}$ of local anaesthetic $(0.5 \%$ bupivacaine with 1 in 100000 adrenaline) was infiltrated subcutaneously. The ellipse of redundant skin was then excised with a blade and scissors.

The anterior tarsal surface was exposed by separating it from the anterior lamella of the eyelid. The pretarsal orbicularis muscle was separated from the overlying skin and care was taken not to perforate or damage the skin. Dissection was continued until the lash roots were just visible (Fig. 2). The pretarsal orbicularis muscle was then excised. Lash eversion was seen immediately when the anterior lamella was repositioned onto the tarsal
C.T. Choo

C.M.L. Chan

K.S. Fong

Singapore National Eye Centre

Dr C.T. Choo, FRCSE, FRCOphth Singapore National Eye Centre

11, 3rd Hospital Avenue Singapore 168751 


\begin{tabular}{|c|c|c|c|c|c|c|c|c|}
\hline \multirow[b]{3}{*}{$\begin{array}{l}\text { Patient } \\
\text { no. }\end{array}$} & \multirow[b]{3}{*}{ Sex/Age/Race } & \multirow[b]{3}{*}{ Symptoms } & \multicolumn{3}{|c|}{ Signs } & \multirow[b]{3}{*}{ Operation } & \multirow[b]{3}{*}{$\begin{array}{c}\text { Follow-up } \\
\text { (months) }\end{array}$} & \multirow[b]{3}{*}{ Lash eversior } \\
\hline & & & \multicolumn{2}{|c|}{ Lash-cornea touch } & \multirow[b]{2}{*}{ PEE } & & & \\
\hline & & & Upgaze & $\begin{array}{l}\text { Primary } \\
\text { position }\end{array}$ & & & & \\
\hline 1 & $\mathrm{~F} / 14 / \mathrm{ch}^{\mathrm{a}}$ & Ocular irritation & + & - & - & R/L ALR & 22 & + \\
\hline 2 & $\mathrm{M} / 28 / \mathrm{ch}^{\mathrm{a}}$ & Ocular irritation & + & - & - & R/L ALR & 6 & + \\
\hline 3 & $\mathrm{M} / 21 / \mathrm{ch}$ & Skin droopy & + & + & + & R/L ALR & 6 & + \\
\hline 4 & $\mathrm{M} / 23 / \mathrm{ch}$ & Single lid & + & - & - & R/L ALR & 6 & + \\
\hline 5 & $\mathrm{M} / 5 / \mathrm{ch}^{\mathrm{a}, \mathrm{b}}$ & Eye rubbing & + & + & + & R/L ALR & 18 & + \\
\hline 6 & $\mathrm{M} / 22 / \mathrm{ch}^{\mathrm{a}}$ & Ocular irritation & + & - & + & R/L ALR & 6 & + \\
\hline 7 & $\mathrm{M} / 8 / \mathrm{ch}^{\mathrm{b}}$ & Eye rubbing & + & - & + & R/L ALR & 11 & + \\
\hline 8 & $\mathrm{~F} / 22 / \mathrm{ch}$ & Ocular irritation & + & - & - & R/L ALR & 11 & + \\
\hline 9 & $\mathrm{M} / 23 / \mathrm{ch}^{\mathrm{a}}$ & Ocular irritation & + & + & + & R/L ALR & 15 & + \\
\hline 10 & $\mathrm{M} / 7 / \mathrm{ch}$ & Ocular irritation, discharge & + & + & + & R/L ALR & $\begin{array}{r}11 \mathrm{~L} \mathrm{go} \\
\text { at }\end{array}$ & $\begin{array}{l}\mathrm{d}, \mathrm{R} \text { recurred } \\
\text { months }\end{array}$ \\
\hline 11 & $\mathrm{M} / 18 / \mathrm{ch}^{\mathrm{a}}$ & Photophobia, tearing & + & + & + & R/L ALR & $\begin{array}{l}\mathrm{L} \text { recurr } \\
\mathrm{R} \text { recurr }\end{array}$ & $\begin{array}{l}d \text { at } 1 \text { month, } \\
d \text { at } 3 \text { months }\end{array}$ \\
\hline 12 & $\mathrm{M} / 20 / \mathrm{ch}$ & Lash ptosis & + & + & - & R/L ALR & 6 & + \\
\hline 13 & $\mathrm{M} / 21 / \mathrm{ch}^{\mathrm{a}}$ & Ocular irritation, photophobia & + & + & + & R/L ALR & 6 & + \\
\hline 14 & $\mathrm{M} / 19 / \mathrm{ch}$ & Ptosis & + & + & + & R/L ALR & 7 & + \\
\hline
\end{tabular}

ch, Chinese; PEE, punctate epithelial erosion; ALR, anterior lamellar reposition; R, right; L, left.

${ }^{\mathrm{a}}$ Case referred as entropion.

${ }^{\mathrm{b}}$ Having lower lid epiblepharon.

plate (Fig. 3). The skin crease was formed by suturing the deep layers of the upper and lower wound edges to the tarsus with $7 / 0$ Vicryl. Skin closure was completed with interrupted $7 / 0$ silk (Fig. 3). These silk sutures were removed at 1 week.

Patients were seen 1 day, 1 week, 1 month, 3 months and 6 months post-operatively. They were followed up

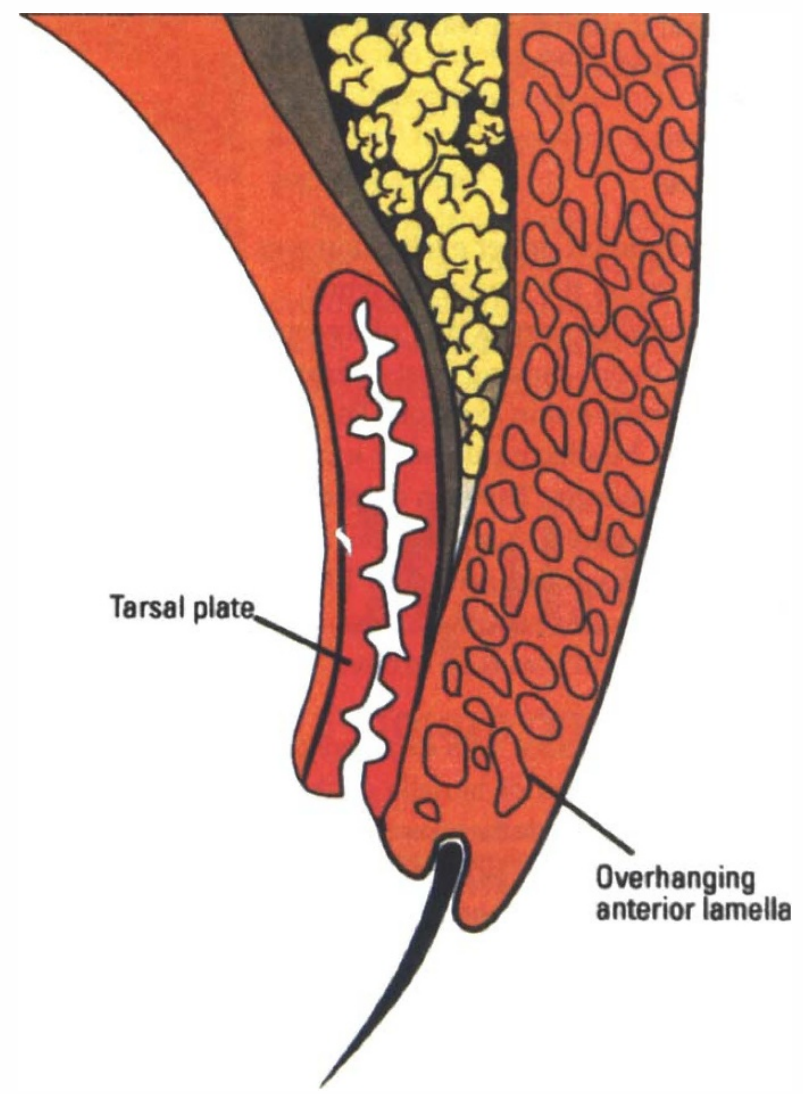

Fig. 1. Lash inversion in upper lid epiblepharon. for a minimum of 6 months (range 6-22 months). Good lash eversion and absence of lash-globe contact had to be achieved before the surgery was considered a success.

\section{Results}

Twenty-eight eyelids of 14 patients with upper eyelid epiblepharon complicated by lash-globe contact were seen and managed surgically. Twelve patients were male and 2 were female. The patients' average age was 18

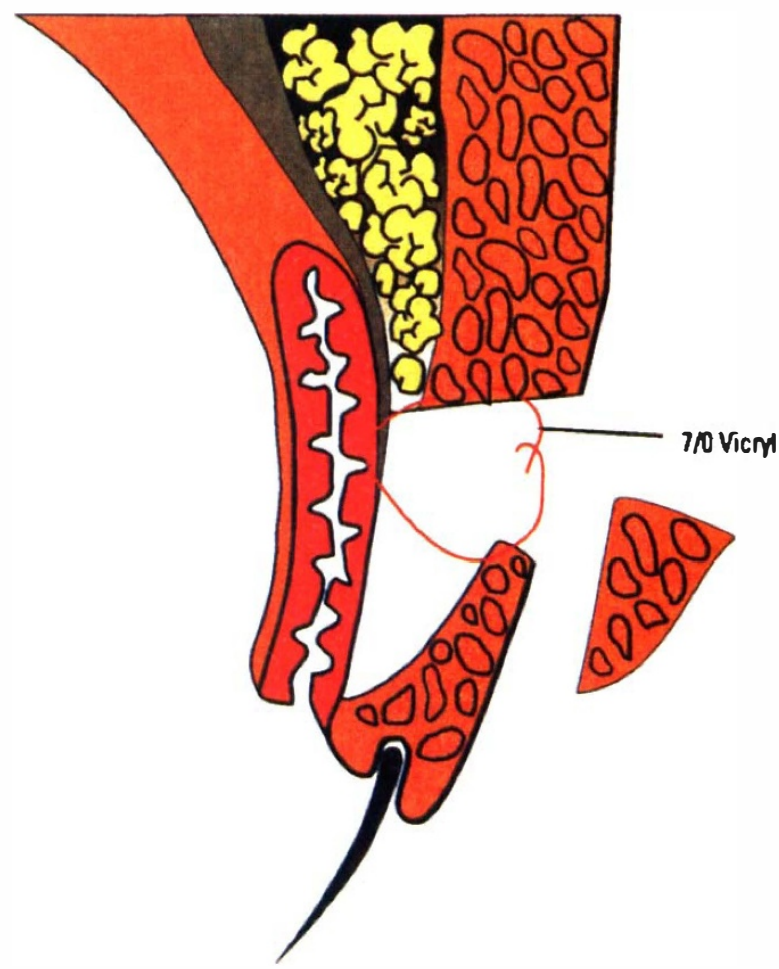

Fig. 2. Pretarsal orbicularis debulking after skin excision. 


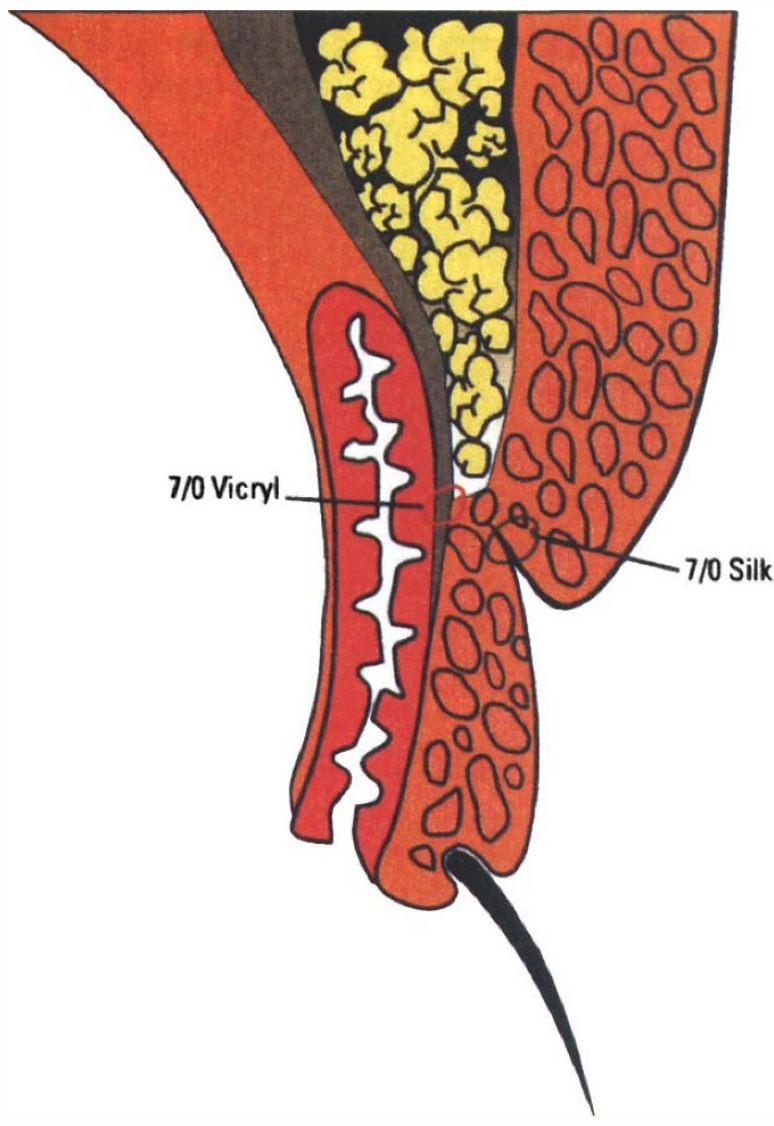

Fig. 3. Lash eversion after anterior lamellar repositioning.

years (range 5-28 years). All the patients were Chinese. Seven patients were initially diagnosed as having upper lid entropion by general ophthalmologists before being referred to our clinic for surgery. They all had lash-cornea touch on upgaze but only 8 of them had it in the primary position as well. Punctate epithelial erosions were seen in 9 patients. Two patients had coexisting lower lid epiblepharon. This was also managed surgically using a similar technique (Table 1).

Surgery was successful in 25 of 28 eyelids (89\%). The follow-up period was 6-22 months. All pre-operative corneal punctate epithelial erosions had healed by the seventh post-operative day and patients reported significant symptomatic relief.

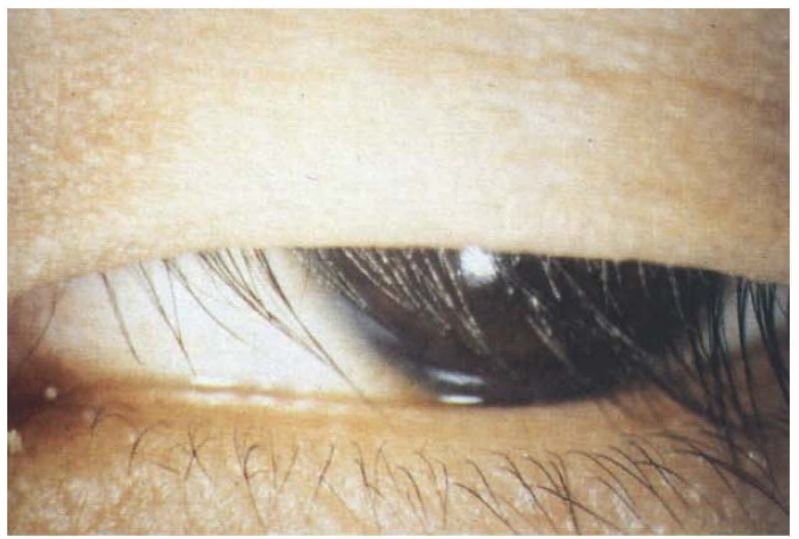

Fig. 4. Case 11. Left upper lid epiblepharon with trichiasis.

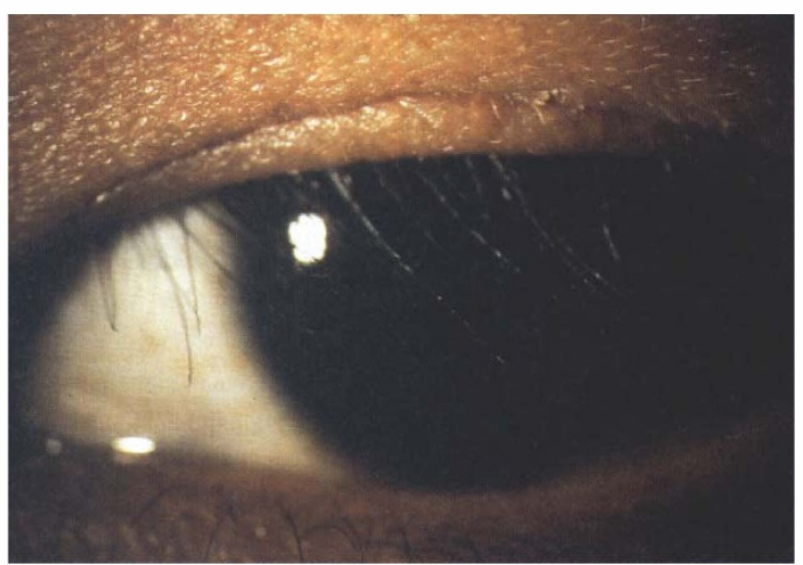

Fig. 5. Case 11. Recurrence of trichiasis 1 month after surgery.

Two patients ( 3 eyelids) had a recurrence. One patient (case 11) had recurrence of trichiasis in the left upper lid at 1 month post-operatively (Fig. 5) and recurrence in the right upper lid at 3 months post-operatively. Repeat surgery was performed on his left upper lid with further skin excision and debulking of the pretarsal orbicularis (Fig. 6). No recurrence was observed after 7 months of follow-up. Repeat surgery was not done for his right upper lid as this side was asymptomatic. Another patient's left upper lid (case 10) had recurrence of lash-cornea touch but repeat surgery was not performed because he had no symptoms.

\section{Discussion}

Upper eyelid epiblepharon refers to the presence of an exaggerated skin fold running horizontally across the upper lid. It has been described as a rare, congenital deformity in Caucasians which is transmitted in a dominant manner. ${ }^{1}$ The aetiology of upper lid epiblepharon has been attributed to a Z-shaped kink in the orbicularis fibres or the absence of levator aponeurotic attachment to the orbicularis and skin. In severe cases it is associated with secondary trichiasis and punctate epithelial erosions sufficient to cause photophobia and a decrease in visual acuity.

Epiblepharon, though uncommon in Caucasians, is frequently encountered in Orientals. They have a lower

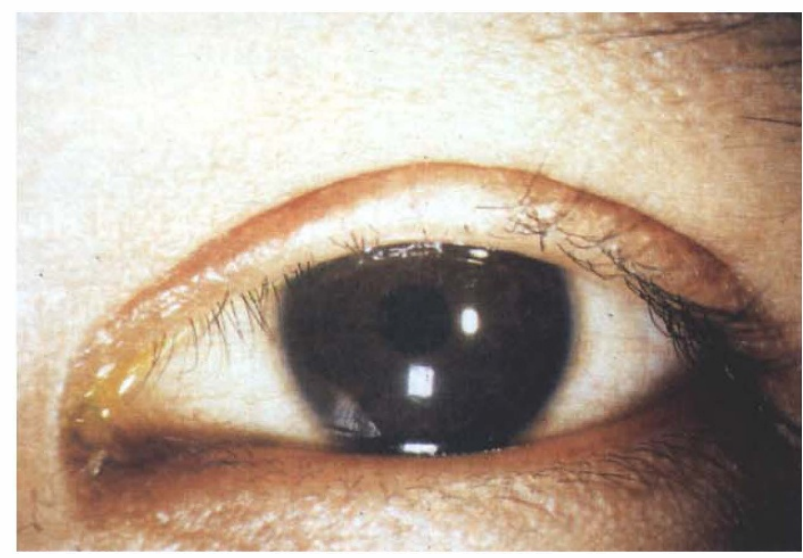

Fig. 6. Case 11. Left upper lid 7 months after repeat surgery. 
insertion of the orbital septum on the aponeurosis which results in a more inferior extension of the orbital fat and a poor attachment of the levator aponeurosis to the subcutaneous tissue. Together with an abundant pretarsal orbicularis and inferior extension of the brow fat pad onto the pretarsal space, a poor/absent skin crease results. ${ }^{4}$ These features are exaggerated in upper lid epiblepharon. Oriental upper lid epiblepharon with lash-cornea touch is commonly seen in our clinical practice. It is quite often misdiagnosed as upper lid entropion; however, its tarsal position, tarsoconjunctival surface and lid margin are all normal. In our series of 14 patients reviewed, 7 were initially diagnosed in the general clinic as having upper lid entropion. From the anatomical viewpoint it is therefore reasonable that only the anterior lamella of the lid overhanging the lid margin and causing the lash inversion should be excised and repositioned.

Many techniques to correct lower lid epiblepharon have been described. These include lid bracing sutures, ${ }^{5}$ buried sutures, skin resection, ${ }^{6}$ a Hotze type procedure, a Jones procedure or its modification. ${ }^{7-10}$ However, reports on the correction of upper lid epiblepharon are sparse. Duke Elder, Sayoc ${ }^{11}$ and Millard ${ }^{12}$ have suggested that the excess skin fold seen in epiblepharon can simply be excised. Johnson, ${ }^{13}$ however, considered upper lid epiblepharon to be an exaggeration of epicanthus tarsalis and recommended a V-Y incision at the inner canthus of the upper lid.

A literature search did not reveal case series of surgical correction of Oriental upper lid epiblepharon. Previous reports on surgery for upper lid epiblepharon have all been anecdotal. In our series, the excision of the skin fold accompanied by pretarsal orbicularis debulking together with skin crease reformation by closure onto the tarsus is a logical way to correct this anomaly. Among the 14 cases performed, 2 also had lower lid epiblepharon which was corrected in a similar fashion albeit without skin crease reformation.

In lower lid epiblepharon no attempt was made to create a skin crease as this was not an essential feature in Oriental patients. ${ }^{10}$ In upper lid epiblepharon we created an upper lid crease at $5 \mathrm{~mm}$ from the lid margin as a low upper lid crease is considered aesthetically pleasing and is acceptable to patients previously without an upper lid skin crease. This is especially so in patients requesting aesthetic upper lid blepharoplasty, commonly called the 'double eyelid operation', in our mainly Oriental population.

In our case series one patient underwent reoperation. Intraoperatively we found that the first surgery had been too conservative in excising the skin and pretarsal orbicularis. After the repeat procedure there has been no recurrence in the 7 months of follow-up.

In conclusion, debulking of the pretarsal orbicularis and skin excision with crease formation is an effective way of treating upper lid epiblepharon with lash ptosis trichiasis. A success rate of $89 \%$ with good lash eversion and good cosmesis has been achieved in Oriental patients with this procedure.

\section{References}

1. Duke-Elder S, editor. System of ophthalmology, vol 3, part 2. London: Henry Kimpton, 1964:857-9.

2. Karlin DB. Congenital entropion, epiblepharon and atimongoloid obliquity of the palpebral fissure. Am J Ophthalmol 1960;50:487-93.

3. Johnson CC. Epiblepharon. Am J Ophthalmol 1968;66:11720-75.

4. Doxanas MT, Anderson RL. Clinical orbital anatomy. Baltimore: Williams \& Wilkins, 1984:chap 4.

5. Quickert MH, Wilkes DI, Dryden RM. Nonincisional correction of epiblepharon and congenital entropion. Arch Ophthalmol 1983;101:778-81.

6. Hayasaka S, Noda S, Setogawa T. Epiblepharon with inverted eyelashes in Japanese children. II. Surgical repairs. Br J Ophthalmol 1989;73:128-30.

7. Hotz FC. A new operation for entropion and trichiasis. Arch Ophthalmol 1879;8:249-63.

8. O'Donnell BA, Collin JRO. Congenital lower eyelid deformity with trichiasis (epiblepharon and entropion). Aust NZ J Ophthalmol 1994;22:33-7.

9. Collin JRO. A manual of systematic eyelid surgery, 2nd ed. Edinburgh: Churchill Livingstone, 1989:chap 2.

10. Choo CT. Correction of Oriental epiblepharon by anterior lamellar reposition. Eye 1996;10:545-7.

11. Sayoc BT. Plastic reconstruction of the superior palpebral fold. Am J Ophthalmol 1954;38:556-9.

12. Millard DR Jr. The oriental eyelid and its surgical revision. Am J Ophthalmol 1964;57:646-9.

13. Johnson CC. Epicanthus and epiblepharon. Arch Ophthalmol 1978;96:1030-3. 